\title{
The Prediction and Function of Neoantigen in Oncobiology
}

\author{
Mengjie Cui \\ College of Life Science, University of Chinese Academy of Science, Beijing 101408 China \\ cuimj96@qq.com
}

Keywords: neoantigen, tumor immunology, mutation, prediction, immunotherapy.

\begin{abstract}
Recent clinical data clearly demonstrated that patient's own immune system has great potential in controlling the progression of many human cancers. Neoantigen is the foundation of tumor immunology for it is the "marker" for mature autologous $\mathrm{T}$ lymphocytes to distinguish tumor cells and normal cells. So identification of neoantigen is an important work in both fundamental studies and clinical immunotherapies. Neoantigen is patient-specific, it is derived from tumor genome somatic mutations in protein coding region. Identification of neoantigen is a laborious work before, but recent technological innovations have made this measurement easier and faster, though still not perfect. Now, numerous neoantigen based clinical trials are being carried out around the world by both academic institutes and companies, some have achieved exciting results.
\end{abstract}

\section{Introduction}

Human tumor cells express antigenic peptides that derived from genome somatic mutations can be recognized by mature autologous $\mathrm{T}$ cells. Enhancement of such immune activity, especially $\mathrm{T}$ lymphocytes' anti-tumor activity, by checkpoint inhibitor antibody, adoptive cell therapy (ACT) or other being-developed novel approaches, can potentially lead to cancer regression in patients with advanced disease [1]. Neoantigen is the foundation of tumor immunology for it is the "biomarker" for autologous mature T lymphocytes to distinguish tumor cell and normal cells [2]. So identification of neoantigen is a pivotal work in both fundamental studies and clinical immunotherapies. With the fast development of next generation sequencing (NGS) and mass spectrometry technology, neoantigens can be indirectly predicted from genome research by generating high-throughput sequencing data or directly identified by LC-MS/MS in an unbiased manner [3, 4].

\section{The "mutanome" of Tumor}

Tumor genome bears numerous somatic mutations due to the imperfect DNA mismatch repair mechanisms and its immortality $[2,5]$. With the next generation sequencing (NGS) technology, we can now get a complete map of mutational landscape in individual tumors (the "mutanome"). Of all cancer types, melanoma, non-small-cell lung cancer (NSCLC), colorectal cancer and bladder cancer have high somatic mutation burdens [6], while mutation rate of some cancers, such as Ewing sarcoma and prostate cancer, is comparatively low [7]. Of these mutations, some occurred on the coding region of genes, which may result in changes in protein sequences (nonsynonymous mutations). A small fraction of these mutations in protein are called "driver mutation", such as mutations in c-Myc, Kras, EGFR, et.ac, that can render tumor with selective growth advantage over normal cells, they are responsible for the initiation or progression of the tumors; the others are called "passenger mutation", which nearly have no influences on the development and progression of cancers [2]. It was found that human cancers carry dozens to hundreds of nonsynonymous mutations, and even thousands in melanoma. Studies also demonstrated that shared mutations are extremely rare and the great majority of mutations are randomly generated and patient specific. Clinical results also showed that the mutation rate is closely correlated with the response rate of anti-PD1 checkpoint antibodies in several tumors [8]. 


\section{Neoantigens in Tumor}

These nonsynonymous mutations cause tumors to express mutant proteins that are not expressed by normal cells [9]. A proportion of these mutant protein can be processed into short peptides and presented on the malignant cell surface by forming MHC-peptide complex (MHC is also known as human leukocyte antigen (HLA) in humans), these short peptides that presented only in tumor cells are called neoantigens. These neoantigens may just have one amino acid difference from the native peptide, but $\mathrm{T}$ cells have the ability to distinguish this tiny difference between mutated and nonmutated epitopes [10]. The MHC-neoantigen complex is recognized by CD8+ cytotoxic T cells and CD4+ helper T cells as "non-self" or foreign. In principle, neoantigens are ideal targets for immune system to recognize and attack cancers as they lack expression in normal cells, which will help to avoid the risk of autoimmunity [11]. As mentioned above, somatic mutations are generated randomly, the majority of the neoantigens in human tumors are not shared between patients, so neoantigens are patient-specific, but the immune system has an extremely diverse $\mathrm{T}$ repertoire to recognize these neoantigens [12]. It was demonstrated that tumors with more neoantigen burden have more tumor infiltrating lymphocytes (TILs) and were more likely to clinically benefit from immune checkpoint inhibitors than those with a lower mutation load [13, 14].

Previously, the majority of researches focused on MHC class I restricted neoantigens. With our deeper understanding of CD4 + T lymphocytes' role in antitumor immunity [15], increasing emphasis has been put on MHC class II restricted neoantigens and it has the potential to be a promising therapeutic target [16].

\section{Neoantigen Prediction and Identification}

\subsection{Classical method}

The initial attempts to identify neoantigen in the past two decades mainly using cDNA library screening method, and predominantly in melanoma, due to its high mutation rate. In this method, pooled cDNA library derived from cancer cells is transfected into cell line that stably express MHC molecules, and then co-cultured with $\mathrm{T}$ cells to identify the antigen that can result in $\mathrm{T}$ cell activation, measured mainly through cytokine secretion or 4-1BB up-regulation $[17,18]$. But, this approach is very inefficient, labor-consuming and time-costing. In addition, genes with large transcripts and lowexpression level may be lost in the process of constructing the cDNA library, which will lead to the failure to identify certain mutated antigens.

\subsection{Next generation sequencing based method}

Human genome has approximately 3 billion nucleotides, but only $1 \%$ of these nucleotides code for genes (the exome). To identify neoantigens, sequencing only the exome can dramatically reduce the cost and minimize the complexity of data analysis [19]. Now, whole-exome sequencing (WES) combined with RNA sequencing (RNA-Seq) and epitope prediction method was proved to be an efficient approach $[3,20]$.

First, somatic mutations in coding regions were detected by whole-exome sequencing of tumor and normal tissues. Then, the mutant gene expression level was analyzed by RNA-Seq. Last, prediction of the binding affinity of candidate peptides to MHC molecules. This is the most critical step, for the human HLA molecule is the most polymorphic gene in human genome, the number of registered HLA class I molecules now over 1500 [21]. So, great efforts have been paid to develop the algorithms to accurately predict the binding affinity, several software or website-based tools have been developed now. These methods mainly fall into two categories: MHC-specific, which a method is applied to individual MHC molecule, such as NetMHC [22], SMM [23], SYFPEITHI [24]; and pan-specific, which a single method is applied to nearly all MHC-I molecules, such as NetMHCpan [25] and NetMHCcons [26]. In particular, the pan-specific method is very powerful tool, considering the huge polymorphism of MHC genes [27]. These useful tools greatly improved the researches of neoantigen on tumor immunity and clinical progresses.

But this approach is restricted by the accuracy of MHC binding prediction algorithms, for the complexity of intracellular epitope processing mechanisms. That is to say, the predicted neoantigens, 
even with high affinity to MHC molecules, may not be actually presented in vivo. To overcome this problem, researchers developed a tandem minigene screening strategy to increase the coverage [28]. In 2015, Gerald P. Linette and colleagues used tandem minigene that code for about 7 neoepitopes in one construct as vaccine to boost the anti-tumor immunity in patient with melanoma. Their results demonstrated the neoantigen vaccine broadens the antigenic breadth and clonal diversity of antitumor immunity. [29].

\subsection{Mass spectrometry based method}

The major histocompatibility complex (MHC) molecules that bind antigenic peptides can be isolated, and peptides can be eluted and purified for identification by liquid chromatography and tandem mass spectrometry (LC-MS/MS) based method, which is a very promising way directly identify tumor neoantigens from cancer biopsies now [4].

Currently, the best established methodology is using anti-HLA monoclonal antibody to immunoprecipitation of HLA-peptide complexes from detergent solubilized tumor lysates, followed by epitopes elution from HLA complex, and purification and concentration of the short peptides [30]. In particular, pan-HLA class I or pan-HLA class II monoclonal antibodies can be used, and different HLA allele-specific antibodies are also available. Then, the extracted short peptides are analyzed by LC-MS/MS. At last, the MS data is searched against personalized protein sequence databases through the commonly used search engines like Mascot or MaxQuant. Typically, thousands of antigen peptide can be identified per sample in days. Compared with NGS based approach, LC-MS/MS is an unbiased methodology to comprehensively analyze the repertoire of tumor neoantigens. Of note, neoantigens detected by MS from a tumor sample will be in vivo naturally and well-presented targets [31]. Then, the immunogenicity of the identified neoantigens should be further confirmed by $\mathrm{T}$ lymphocytes functional assays, using $\mathrm{T}$ cell isolated from PBMCs or directly from tumor infiltrating lymphocytes. The valid immunogenic epitopes can be used for personalized antitumor immunotherapy.

To predict neoantigens with more accuracy, Lelia Delamarre and colleagues developed a strategy that combines whole-exome and RNA sequencing data with mass spectrometry to identify neoantigens. Of the 1300 amino acid mutations, $13 \%$ were predicted to bind MHC I molecules, and a small percentage of them was further confirmed by mass spectrometry [32].

\section{Therapeutic Approaches Targeting Patient Specific Neoantigen}

Several approaches for targeting personalized neoantigens have been proposed. Of them, neoantigen specific $\mathrm{T}$ cell adoptive cell therapy and personalized neoantigen vaccines are two most promising methods.

Steven A. Rosenberg at National Cancer Institute is a pioneer in developing effective $\mathrm{T}$ cell based therapy. In 2014, his group generated neoantigen specific CD4 T lymphocytes from a metastatic cholangiocarcinoma, in vitro cultured and transferred back to the patient, the result demonstrated that this treatment successfully induced tumor regression [1, 28, 33]. It was reported recently that neoantigen specific $\mathrm{T}$ cells that generated from healthy donors were able to recognize 11 of 57 predicted neoepitopes across three patients. This may broaden neoantigen-specific $\mathrm{T}$ cell reactivity, and provide a rationale for using "outsourced" $\mathrm{T}$ cells in curing tumor patients with dysfunctional immune system [34].

Another strategy, personalized neoantigen vaccine, designed to use synthesized neoantigen peptides or RNA that coding tandem minigenes to prime and activate DCs, and then to boost T cell immunity targeting the neoantigen expressed by tumors. Although researchers failed many times in developing therapeutic tumor vaccines, two most recent studies showed exciting results in melanoma. Catherine J. Wu and colleagues combined whole exome sequencing, RNA sequencing and in silico prediction to obtain candidate neoantigens, then synthesized them and administered to patients. Results showed that vaccine induced polyfunctional CD4+ and CD8+ T cells targeted $60 \%$ and $16 \%$ of the neoantigens used in all patients. Of 6 treated patients, 4 had no recurrence after vaccination for 2 years, while 2 patients with recurrent disease were treated with anti-PD- 1 antibody (pembrolizumab) and experienced complete tumour regression [35]. Another study used nearly the same strategy to 
predict neoantigens, they used RNA minigenes as neoantigen vaccine and injected percutaneously into inguinal lymph nodes. Results showed that all 13 patients developed $\mathrm{T}$ cell responses against multiple vaccine neoantigens, 8 of them had no radiologically detectable lesions for 12 to 23 months, and another 2 patient also experienced complete response through additional anti-PD1 therapy [36]. In sum, combined neoantigen vaccine with anti-PD1 antibody, researchers observed the duration of the vaccine induced immune responses over more than 2 years and the increasing diversity of the repertoire of neoantigen-specific $\mathrm{T}$ cells.

\section{Conclusion}

Antitumor immunity exists in every tumor patient, though it may be weak or inefficient in the complex tumor environment context, so what we are doing now in essence is to "release the brake" or boost the antitumor activity [37]. Neoantigen is the foundation of tumor immunity, it is the real target that $\mathrm{T}$ lymphocytes can distinguish tumor cells from normal cells. With our deeper understanding of tumor immunology and more clinical data available, the importance of neoantigen in both diagnosis and treatment of cancer is gradually being realized, and in the future, therapeutic strategies based on neoantigen will be extensively studied [38]. Now, two key questions should be solved immediately. First, the neoantigen prediction method. So far, the most useful approach for identifying T cell neoepitopes is MHC-peptide binding affinity prediction, though LC-MS/MS based method is an unbiased way to comprehensively analyze the in vivo repertoire of tumor neoantigens, its sensitivity need to be further improved [39]. Second, the optimal neoantigen-based therapeutic method in clinical trials must be established. Both adoptive cell transfer and neoantigen vaccine are very promising methods [40], some new strategies are also being developed. To maximize antitumor functions, a combination with checkpoint inhibitor, such as anti-PD1 antibody, may be necessary. Actually, based on the clinical data available till now, neoantigen vaccine plus checkpoint inhibitor antibody combination therapy strategy may be the most promising one $[35,36]$.

\section{References}

[1]. Yarchoan, M., et al., Targeting neoantigens to augment antitumour immunity. Nat Rev Cancer, 2017. 17(4): p. 209-222.

[2]. Tran, E., P.F. Robbins, and S.A. Rosenberg, 'Final common pathway' of human cancer immunotherapy: targeting random somatic mutations. Nature Immunology, 2017. 18(3): p. 255262.

[3]. Pritchard, A.L., et al., Exome Sequencing to Predict Neoantigens in Melanoma. Cancer Immunol Res, 2015. 3(9): p. 992-8.

[4]. Polyakova, A., K. Kuznetsova, and S. Moshkovskii, Proteogenomics meets cancer immunology: mass spectrometric discovery and analysis of neoantigens. Expert Rev Proteomics, 2015. 12(5): p. 533-41.

[5]. Wang, R.F. and H.Y. Wang, Immune targets and neoantigens for cancer immunotherapy and precision medicine. Cell Research, 2017. 27(1): p. 11-37.

[6]. Schumacher, T.N. and R.D. Schreiber, Neoantigens in cancer immunotherapy. Science, 2015. 348(6230): p. 69-74.

[7]. Vogelstein, B., et al., Cancer Genome Landscapes. Science, 2013. 339(6127): p. 1546-1558.

[8]. Rizvi, N.A., et al., Mutational landscape determines sensitivity to PD-1 blockade in non-small cell lung cancer. Science, 2015. 348(6230): p. 124-128.

[9]. Schumacher, T.N. and N. Hacohen, Neoantigens encoded in the cancer genome. Curr Opin Immunol, 2016. 41: p. 98-103.

[10]. Bobisse, S., et al., Neoantigen-based cancer immunotherapy. Ann Transl Med, 2016. 4(14): p. 262.

[11]. Coulie, P.G., et al., Tumour antigens recognized by $\mathrm{T}$ lymphocytes: at the core of cancer immunotherapy. Nat Rev Cancer, 2014. 14(2): p. 135-46. 
[12]. Matsushita, H., et al., Cancer exome analysis reveals a T-cell-dependent mechanism of cancer immunoediting. Nature, 2012. 482(7385): p. 400-U149.

[13]. .van Rooij, N., et al., Tumor exome analysis reveals neoantigen-specific T-cell reactivity in an ipilimumab-responsive melanoma. J Clin Oncol, 2013. 31(32): p. e439-42.

[14]. .Riaz, N., et al., The role of neoantigens in response to immune checkpoint blockade. Int Immunol, 2016. 28(8): p. 411-9.

[15]. Flament, H., et al., Modeling the specific CD4+ T cell response against a tumor neoantigen. $\mathbf{J}$ Immunol, 2015. 194(7): p. 3501-12.

[16]. Sun, Z., et al., MHC class II restricted neoantigen: A promising target in tumor immunotherapy. Cancer Lett, 2017. 392: p. 17-25.

[17]. .Ratnoff, W.D., W.W. Brockman, and L.A. Hasty, Immunohistochemical localization of C9 neoantigen and the terminal complement inhibitory protein CD59 in human endometrium. Am J Reprod Immunol, 1995. 34(2): p. 72-9.

[18]. .Tobi, M., et al., Urinary organ specific neoantigen. A potentially diagnostic test for colorectal cancer. Dig Dis Sci, 1995. 40(7): p. 1531-7.

[19]. Patel, A., H.L. Kaufman, and M.L. Disis, Next generation approaches for tumor vaccination. Chin Clin Oncol, 2017. 6(2): p. 19.

[20]. Karasaki, T., et al., Prediction and prioritization of neoantigens: integration of RNA sequencing data with whole-exome sequencing. Cancer Sci, 2017. 108(2): p. 170-177.

[21]. Nielsen, M., et al., NetMHCpan, a method for quantitative predictions of peptide binding to any HLA-A and -B locus protein of known sequence. PLoS One, 2007. 2(8): p. e796.

[22]. .Lundegaard, C., et al., NetMHC-3.0: accurate web accessible predictions of human, mouse and monkey MHC class I affinities for peptides of length 8-11. Nucleic Acids Res, 2008. 36(Web Server issue): $\mathrm{p}$. W509-12.

[23]. .Peters, B. and A. Sette, Generating quantitative models describing the sequence specificity of biological processes with the stabilized matrix method. BMC Bioinformatics, 2005. 6: p. 132.

[24]. .Rammensee, H., et al., SYFPEITHI: database for MHC ligands and peptide motifs. Immunogenetics, 1999. 50(3-4): p. 213-9.

[25]. Nielsen, M. and M. Andreatta, NetMHCpan-3.0; improved prediction of binding to MHC class I molecules integrating information from multiple receptor and peptide length datasets. Genome Med, 2016. 8(1): p. 33.

[26]. .Karosiene, E., et al., NetMHCcons: a consensus method for the major histocompatibility complex class I predictions. Immunogenetics, 2012. 64(3): p. 177-86.

[27]. Robinson, J., et al., The IPD and IMGT/HLA database: allele variant databases. Nucleic Acids Res, 2015. 43(Database issue): p. D423-31.

[28]. Tran, E., et al., Cancer immunotherapy based on mutation-specific CD4+ T cells in a patient with epithelial cancer. Science, 2014. 344(6184): p. 641-5.

[29]. Carreno, B.M., et al., A dendritic cell vaccine increases the breadth and diversity of melanoma neoantigen-specific T cells. Science, 2015. 348(6236): p. 803-808.

[30]. Antwi, K., et al., Proteomic identification of an MHC-binding peptidome from pancreas and breast cancer cell lines. Mol Immunol, 2009. 46(15): p. 2931-7.

[31]. .Bassani-Sternberg, M. and G. Coukos, Mass spectrometry-based antigen discovery for cancer immunotherapy. Curr Opin Immunol, 2016. 41: p. 9-17.

[32]. .Yadav, M., et al., Predicting immunogenic tumour mutations by combining mass spectrometry and exome sequencing. Nature, 2014. 515(7528): p. 572-6.

[33]. Robbins, P.F., et al., Mining exomic sequencing data to identify mutated antigens recognized by adoptively transferred tumor-reactive T cells. Nat Med, 2013. 19(6): p. 747-52.

[34]. Stronen, E., et al., Targeting of cancer neoantigens with donor-derived $\mathrm{T}$ cell receptor repertoires. Science, 2016. 352(6291): p. 1337-41.

[35]. Ott, P.A., et al., An immunogenic personal neoantigen vaccine for patients with melanoma. Nature, 2017. 
[36]. Sahin, U., et al., Personalized RNA mutanome vaccines mobilize poly-specific therapeutic immunity against cancer. Nature, 2017.

[37]. Liu, Y., Neoantigen: A Long March toward Cancer Immunotherapy. Clin Cancer Res, 2016. 22(11): p. 2602-4.

[38]. Lu, Y.C. and P.F. Robbins, Cancer immunotherapy targeting neoantigens. Seminars in Immunology, 2016. 28(1): p. 22-27.

[39]. Bassani-Sternberg, M., et al., Direct identification of clinically relevant neoepitopes presented on native human melanoma tissue by mass spectrometry. Nat Commun, 2016. 7: p. 13404.

[40]. Fritsch, E.F., N. Hacohen, and C.J. Wu, Personal neoantigen cancer vaccines: The momentum builds. Oncoimmunology, 2014. 3: p. e29311. 